is a central $(1, p)$-atom (see [5]) of the space $H A^{p}\left(\mathbb{R}^{n}\right)$. So, by Theorem 3.1 of [5], it follows that

$$
f_{2}(x)=\left\|f_{2}\right\|_{p}|B(0,1)|^{1-1 / p} b(x) \in H A^{p}\left(\mathbf{R}^{n}\right) .
$$

Hence $f(x)=f_{1}(x)+f_{2}(x) \in H A^{p}\left(\mathbf{R}^{n}\right)$, that is, $H K_{p}\left(\mathbb{R}^{n}\right) \cap L_{\text {loc }}^{p}\left(\mathbf{R}^{n}\right) \subset$ $H A^{p}\left(\mathbf{R}^{n}\right)$.

This finishes the proof of Remark.

\title{
References
}

[1] Y. Z. Chen and K. S. La u, On some new classes of Hardy spaces, J. Funct. Anal, 84 (1989), 255-278.

[2] C. Fefferman and E. M. Stein, $H^{p}$ spaces of several variables, Acta Math. 129 (1972), 137-193.

[3] M. Frazier and B. Jawerth, The $\varphi$-transform and applications to distribution spaces, in: Function Spaces and Applications, Lecture Notes in Math. 1302, Springer,
1988, 223-246.

[4] - - A discrete transform and decompositions of distribution spaces, J. Funct. Anal. 93 (1990), 34-170.

[5] J. García-Cuerva, Hardy spaces and Beurling algebras, J. London Math. Soc. (2) 39 (1989), 499-513.

[6] C. Herz, Lipschitz spaces and Bernstein's theorem on absolutely convergent Fourier transform, J. Math. Mech. 18 (1968), 283-324.

[7] D. S. Kurtz, Littlewood-Paley operators on BMO, Proc. Amer. Math. Soc. 99 (1987), 657-666.

[8] S. Lu and D. Yang, Some new Hardy spaces associated with the Herz spaces, to appear.

[9] A. Torchinsky, Real-variable Methods in Harmonic Analysis, Academic Press, New York 1986.

[10] D. Yang, The boundedness of generalized Littlewood-Paley functions, Chinese Ann. Math. Ser. A 12 (1991), 736- -744.

DEPARTMENT OF MATHEMATICS

BEIJING NORMAL UNIVERSITY

100875 BEIJING, P.R. CHINA

\section{Sur les espaces de Fréchet ne contenant pas $c_{0}$}

\author{
par
}

\author{
X. FERNIQUE (Strasbourg et Chapel Hill, N.C.)
}

Sommaire. Soit $E$ un espace de Fréchet séparable ne contenant pas $c_{0}$; soit de plus $\left(X_{n}\right)$ une suite symétrique de vecteurs aléatoires à valeurs dans $E$. Alors si la série de Fourier aléatoire $\sum X_{n} \exp \left(i\left\langle\lambda_{n}, t\right\rangle\right), t \in \mathbf{R}^{d}$, a p.s. ses sommes partielles localement uniformément bornées dans $E$, nécessairement elle converge p.s. uniformément sur tout compact de $\mathbb{R}^{d}$ vers une fonction aléatoire à valeurs dans $E$ et à trajectoires continues.

\section{Introduction, notations, énoncé}

1.1. Dans deux papiers récents, $M$. Talagrand et $X$. Fernique étudient respectivement les séries de Fourier gaussiennes à valeurs dans un espace de Banach séparable $E$ ([8]) et les fonctions aléatoires gaussiennes stationnaires sur $\mathbf{R}^{d}$ à valeurs dans un espace lusinien quasi-complet $E\left([3],\left[3^{\prime}\right]\right)$. L'un et l'autre montrent que si les éléments aléatoires qu'ils étudient sont p.s. localement bornés dans $E$ et si $E$ ne contient pas $c_{0}$, alors ces éléments ont des propriétés de continuité. Dans ces études qui prolongent les plus anciens résultats de Hoffmann-Jørgensen ([4]) et de Kwapień ([6]) sur les espaces de Banach ne contenant pas $c_{0}$, le caractère gaussien semble important. On se propose de montrer ici qu'en fait l'analyse des énoncés de [8] et des méthodes d'étude de [3] permet de supprimer toute hypothèse gaussienne et même tout calcul gaussien, au prix d'une restriction sur les espaces considérés par rapport à $[\mathfrak{3}],\left[\mathfrak{3}^{\prime}\right]$.

Dans tout ce travail, $E$ désignera un espace de Fréchet séparable (complexe); on notera $\left(\lambda_{n}\right)$ une suite d'éléments de $\mathbf{R}^{d}$ et $\left(X_{n}\right)$ une suite symétrique de vecteurs aléatoires à valeurs dans $E$; on supposera que l'espace d'épreuves est complet et on posera $S_{0}=\{\mathbf{Q} \cap[0,1]\}^{d}, S=\mathbf{Q}^{d}$. On dira que $E$ contient $c_{0}$ si :

(1.1.1) Il existe une suite $\left(x_{n}\right)$ contenue dans $E$ telle que pour toute suite

1991 Mathematics Subject Classification: 60B11, 42A32, 42Bxx.

Recherche financée en partie par Air Force Office of Scientific Research Contract No. AFOSR-91-0030 à l'Université de Caroline du Nord à Chapel Hill. 
numérique $\left(a_{n}\right)$, on ait : $\sum a_{n} x_{n}$ converge dans $E$ si et seulement si $\left(a_{n}\right)$ appartient à $c_{0}$.

Dans ces conditions, on se propose de comparer les deux propriétés suivantes :

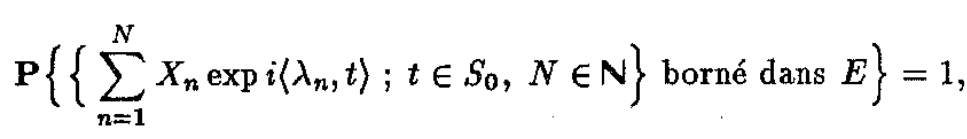

$$
\mathbf{P}\left\{\sum_{n=1}^{\infty} X_{n} \exp i\left\langle\lambda_{n}, t\right\rangle, t \in[0,1]^{d}\right.
$$

$$
\text { converge uniformément dans } E\}=1 \text {. }
$$

On remarquera que ces deux propriétés sont trivialement équivalentes si la suite $\left(\lambda_{n}\right)$ est bornée puisque sous cette condition et $\mathrm{si}(1.1 .2)$ est vérifiée, la série $\sum_{n=1}^{\infty} X_{n} \exp i\left\langle\lambda_{n}, t\right\rangle, t \in[0,1]^{d}$, a presque sûrement pour somme une fonction analytique entière et donc continue vers laquelle elle converge uniformément.

1.2. Le résultat présenté ici est énoncé dans le théorème suivant :

THÉORÈm E 1.2. (a) On suppose $d=1$; soient $E$ un espace de Fréchet séparable contenant $c_{0}$ et $\left(\lambda_{n}\right)$ une suite positive non bornée; il existe alors une suite symétrique $\left(X_{n}\right)$ de vecteurs aléatoires à valeurs dans $E$ vérifiant la propriété (1.1.2) sans vérifier la propriété (1.1.3).

(b) Inversement, on suppose d arbitraire; soient $E$ un espace de Fréchet séparable ne contenant pas $c_{0}$ et $\left(\lambda_{n}\right)$ une suite dans $\mathbf{R}^{d}$; alors toute suite symétrique $\left(X_{n}\right)$ de variables aléatoires à valeurs dans $E$ vérifiant la propriété (1.1.2) vérifie aussi la propriété (1.1.3).

Corollaire 1.2.1. Soit $E$ un espace de Fréchet séparable ne contenant pas $c_{0} ;$ soit de plus $\left(X_{n}\right)$ une suite indépendante de variables aléatoires $\dot{a}$ valeurs dans $E$; on suppose que

$$
\mathbf{P}\left\{\left\{\sum_{n=1}^{N} X_{n} \exp i\left\langle\lambda_{n}, t\right\rangle ; t \in S_{0}, N \in \mathbf{N}\right\} \text { borné dans } E\right\}=1 \text {. }
$$

Alors il existe une suite non aléatoire $\left(a_{n}\right)$ d'éléments de $E$ tels que :

(i) l'ensemble $\left\{\sum_{n=1}^{N} a_{n} \exp i\left\langle\lambda_{n}, t\right\rangle ; t \in S_{0}, N \in \mathbf{N}\right\}$ soit borné dans $E$,

(ii) $\mathbf{P}\left\{\sum_{n=1}^{\infty}\left[X_{n}-a_{n}\right] \exp i\left\langle\lambda_{n}, t\right\rangle ; t \in[0,1]^{d}\right.$, converge uniformément dans $E\}=1$.

R e m a r q u e 1.2.2. Le théorème énoncé ci-dessus généralise et relie entre eux le théorème de Billard sur la convergence des séries de Fourier réelles à coefficients symétriques aléatoires et le théorème de Hoffmann-Jørgensen et Kwapień ([4] et [6]) sur les séries de vecteurs aléatoires à valeurs dans les espaces de Banach; les démonstrations que nous présenterons ci-dessous n'utiliseront pas ces théorèmes dont elles fourniront d'ailleurs de nouvelles preuves.

Le schéma d'étude utilisé n'autorisera pas à étendre l'étude aux espaces lusiniens quasi-complets plus généraux (cf. [3]). Par contre, il permettrait aussi bien d'étudier des séries de Fourier sur un groupe localement compact abélien arbitraire (cf. [8]),

Dans le paragraphe 2, nous présentons les résultats techniques nécessaires pour la preuve du théorème que nous développerons ensuite au paragraphe 3.

\section{Eléments techniques pour la preuve}

2.1. Dans tout ce paragraphe, on note $\left(\varepsilon_{n}\right)$ une suite de v.a. de Rademacher indépendantes et $\left(x_{n}\right)$ une suite d'éléments de $E$; on suppose que l'espace $E$ ne contient pas $c_{0}$ et que

$$
\forall n \in \mathrm{N}, \quad X_{n}=\varepsilon_{n} x_{n} ;
$$

pour alléger l'écriture, on posera d'ailleurs :

$$
\forall t \in \mathbf{R}^{d}, \forall n \in \mathbf{N}, \quad X_{n}(t)=X_{n} \exp i\left\langle\lambda_{n}, t\right\rangle .
$$

On suppose aussi que la propriété (1.1.2) est vérifiée. Dans ces conditions, on a

Proposition 2.1. La propriété (1.1.2) implique que

(2.1.1) Il existe une suite $\left(n_{k}\right)$ d'entiers croissant vers l'infini telle que

$$
\mathbf{P}\left\{\forall t \in[0,1]^{d}, \sum_{k=0}^{\infty}\left\{\sum X_{n}(t) ; n_{k} \leq n<n_{k+1}\right\} \text { converge }\right\}=1 .
$$

En particulier, elle implique aussi

$$
\forall t \in[0,1]^{d}, \quad \mathbf{P}\left\{\sum_{n=0}^{\infty} X_{n}(t) \text { converge }\right\}=1 .
$$

Démonstration. Les propriétés d'intégrabilité des séries de Rademacher et les propriétés des parties bornées des espaces de Fréchet séparables ([2], théorème 1.8) montrent que sous les hypothèses indiquées, il existe une partie symétrique convexe fermée et bornée (non aléatoire) $B$ de $E$ telle que

$$
E \sup _{m \in N} \sup \left\{N_{B}\left[\sum_{n=1}^{m} X_{n}(t)\right] ; t \in[0,1]^{d}\right\}<\infty \text {. }
$$


Ceci suffit pour construire une suite non aléatoire $\left(n_{k}\right)$ d'entiers croissant vers l'infini telle que la série de terme général

$$
\begin{aligned}
2^{k+1} \mathbf{P}\left\{\sup _{m \in N} \sup \{\right. & \left.N_{B}\left[\sum_{n=1}^{m} X_{n}(t)\right] ; t \in[0,1]^{d}\right\} \\
& \left.>\sup _{m \leq n_{k}} \sup \left\{N_{B}\left[\sum_{n=1}^{m} X_{n}(t)\right] ; t \in[0,1]^{d}\right\}+2^{-k}\right\}
\end{aligned}
$$

soit convergente. Notons alors $J$ le couple $\{-1,+1\}$; pour tout entier $k$ et tout $\eta \in J^{k}$, posons

$$
\begin{aligned}
\mu_{k}(\eta)=\sup _{m \leq n_{k}} \sup \left\{N _ { B } \left[\sum _ { j = 1 } ^ { k } \eta _ { j } \left[\sum X_{n}(t) ;\right.\right.\right. \\
\left.\left.\left.n_{j} \leq n<(m+1) \wedge n_{j+1}\right]\right], t \in[0,1]^{d}\right\} ;
\end{aligned}
$$

les propriétés de symétrie de la loi de la suite $\left(X_{n}\right)$ montrent que

$$
\sum_{k=0}^{\infty}\left[\sum_{\eta \in J^{k+1}} \mathbf{P}\left\{\mu_{k+1}(\eta)>\mu_{k}(\eta)+2^{-k}\right\}\right]<\infty
$$

le lemme de Borel-Cantelli implique donc qu'il existe un ensemble presque sûr $\Omega_{0}$ tel que

$$
\forall \omega \in \Omega_{0}, \exists k_{0} \in \mathbf{N}: \forall k \geq k_{0}, \forall \eta \in J^{k+1}, \quad \begin{aligned}
& \mu_{k+1}(\eta) \leq \mu_{k}(\eta)+2^{-k} \\
& \\
& \mu_{k+1}(\eta) \leq \mu_{k_{0}}(\eta)+1 .
\end{aligned}
$$

Ceci signifie en particulier que pour tout $\omega \in \Omega_{0}$ et tout $t \in[0,1]^{d}$, la suite $\left(x_{k}\right)$ définie par

$$
x_{k}=\left[\sum X_{n}(\omega, t) ; n_{k} \leq n<n_{k+1}\right]
$$

vérifie la propriété suivante :

L'ensemble $\left\{\sum_{n=1}^{N} \eta_{n} x_{n} ; \eta \in\{-1,+1\}^{N}, N \in \mathrm{N}\right\}$ est un ensemble borné dans $E$.

L'espace de Fréchet $E$ étant complet, on en déduit de la même façon que dans les espaces de Banach que la suite $\left(x_{k}\right)$ vérifie aussi :

$$
\text { Pour tout }\left(a_{n}\right) \in c_{0}, \sum a_{n} x_{n} \text { converge dans } E \text {. }
$$

Puisque $E$ ne contient pas $c_{0}$, on en déduit que la série $\sum x_{k}$ converge; c'est la première conclusion de la proposition. La seconde partie s'en déduit immédiatement à partir des inégalités de Lévy appliquées pour chaque $t$ fixé dans $[0,1]^{d}$ aux restes de la série $\sum_{n=0}^{\infty} X_{n}(t)$; on a en effet

$$
\begin{aligned}
\forall t \in[0,1]^{d}, \forall h> & 0, \quad \mathbf{P}\left\{\lim _{n \rightarrow \infty} \sup _{m \geq n}\left|\sum_{j=n}^{m} X_{j}(t)\right|>h\right\} \\
& \leq 2 \lim _{p \rightarrow \infty} \mathbf{P}\left\{\left|\sum_{k=p}^{\infty}\left\{\sum X_{n}(t) ; n_{k} \leq n<n_{k+1}\right\}\right|>h\right\}=0,
\end{aligned}
$$

la proposition est donc démontrée.

Sous l'hypothèse (1.1.2), la proposition 2.1 permet de définir des fonctions aléatoires $X$ et $R_{k}, k \in \mathrm{N}$, sur $\mathbf{R}^{d}$ à valeurs dans $E$ en posant

$$
X(\omega, t)= \begin{cases}\sum_{n=1}^{\infty} X_{n}(\omega, t) & \text { si la série converge dans } E \\ 0 & \text { sinon, }\end{cases}
$$

et de la même manière

$$
\forall k \in \mathrm{N}, \quad R_{k}(\omega, t)= \begin{cases}\sum_{n=k}^{\infty} X_{n}(\omega, t) & \text { si la série converge dans } E, \\ 0 & \text { sinon. }\end{cases}
$$

Proposition 2.2. La fonction aléatoire $X$ est continue en probabilité.

Dém on stration. Les propriétés de contraction des séries de Rademacher ([7], théorème 2.4.9) montrent que pour tout $t \in \mathbf{R}^{d}$ et toute semi-norme continue $N$ sur $E$, on a

$$
\forall k \in \mathbf{N}, \quad \mathbf{E} N\left[R_{k}(t)\right] \leq 2 \mathbf{E} N\left[R_{k}(0)\right] ;
$$

la conclusion résulte donc de la continuité en probabilité des sommes partielles.

Proposition 2.3. La propriété (1.1.3) est équivalente à la propriété suivante:

$$
\begin{aligned}
& \text { Pour toute semi-norme continue } N \text { sur } E \text {, on } a \\
& \qquad \mathbf{P}\left\{\limsup _{t \rightarrow 0, t \in S} N[X(t)-X(0)]=0\right\}=1 .
\end{aligned}
$$

La démonstration de la proposition 2.3 utilisera les propriétés d'oscillation des séries de Rademacher; on trouverait une démonstration assez voisine d'une propriété apparemment équivalente dans [1]. Le résultat de [1] reste malheureusement inutilisable ici puisqu'il exige que $X$ soit séparable et continu en probabilité; sans doute $X$ est-il continu en probabilité, mais cette seule propriété ne permet d'en construire une modification séparable que si l'espace $E$ est de dimension finie; il n'est pas immédiat d'ailleurs que la propriété (2.3.1) implique que les trajectoires de $X$ aient les propriétés de compacité qui permettraient ([2], théorème 1.4.3) d'en construire une modification séparable même en dimension infinie; enfin, il ne suffirait pas, 
pour pouvoir appliquer le résultat de [1], d'utiliser une modification pseudoséparable au sens du théorème 4.1.8 de [2]. Le lemme ci-dessous utilise une notion d'oscillation adaptée à la non-séparabilité de $X$; il en donne aussi, à partir de la seule loi de $X$, un calcul effectif qui sera nécessaire dans la suite.

LEMME 2.3.2. Pour toute semi-norme continue $N$ sur $E$, il existe un ensemble presque sûr $\Omega_{0}$ et une application non aléatoire $w$ de $\mathbb{R}^{d}$ dans $\mathbb{R}^{+}$ tels que pour tout $\omega \in \Omega_{0}$ et $t \in \mathbf{R}^{d}$,

$\lim _{\varepsilon \rightarrow 0}\left[\sup \left\{N\left[X(\omega, s)-X\left(\omega, s^{\prime}\right)\right] ;|t-s| \vee\left|t-s^{\prime}\right| \leq \varepsilon,\left\{s, s^{\prime}\right\} \subset S\right\}\right]=w(t)$, et pour tout $t \in \mathbf{R}^{d}$,

$$
w(t)=\lim _{\varepsilon \rightarrow 0} \mathbf{E} \sup \left\{N\left[X(s)-X\left(s^{\prime}\right)\right] ;|t-s| \vee\left|t-s^{\prime}\right| \leq \varepsilon,\left\{s, s^{\prime}\right\} \subset S\right\} .
$$

Démonstration du lemme 2.3.2. Nous fixons une semi-norme continue $N$ sur $E$; pour toute fonction $f$ définie sur $S$ à valeurs dans $E$, nous posons pour tout $t \in \mathbf{R}^{d}$,

$$
\begin{aligned}
V(f, t, u)= & \lim _{\varepsilon \rightarrow 0} \sup \left\{N\left[f(s)-f\left(s^{\prime}\right)\right] ;|t-s| \vee\left|t-s^{\prime}\right| \leq u\right. \\
& \left.\left|s-s^{\prime}\right| \leq \varepsilon,\left\{s, s^{\prime}\right\} \subset S\right\} \\
V(f, t)= & \lim _{\varepsilon \rightarrow 0} \sup \left\{N\left[f(s)-f\left(s^{\prime}\right)\right] ;|t-s| \vee\left|t-s^{\prime}\right| \leq \varepsilon,\left\{s, s^{\prime}\right\} \subset S\right\}
\end{aligned}
$$

on a donc $V(f, t)=\lim _{u \rightarrow 0} V(f, t, u)$. Il existe alors un ensemble presque sûr $\Omega_{0}$ tel que

$$
\forall \omega \in \Omega_{0}, \forall s \in S, \quad X(\omega, s)=\sum_{n=1}^{\infty} X_{n}(\omega, s),
$$

et aussi

$$
\forall \omega \in \Omega_{0}, \forall s \in S, \forall k \in \mathrm{N}, \quad R_{k}(\omega, s)=\sum_{n=k}^{\infty} X_{n}(\omega, s)
$$

on a immédiatement du fait de l'uniforme continuité des exponentielles

$$
\forall \omega \in \Omega_{0}, \forall t \in \mathbf{R}^{d}, \forall u>0, \forall k \in \mathrm{N}, \quad V(X(\omega), t, u)=V\left(R_{k}(\omega), t, u\right)
$$

il en résulte que pour $t$ et $u$ fixés, la variable aléatoire $\omega \rightarrow V(X(\omega), t, u)$ est une variable aléatoire terminale pour la suite indépendante $\left(\varepsilon_{n}\right)$, c'est donc une variable aléatoire dégénérée; le théorème de convergence monotone et les propriétés d'intégrabilité des séries de Rademacher fixent d'ailleurs sa valeur presque sûre : il existe un ensemble presque sûr $\Omega_{1}$ tel que

$$
\forall \omega \in \Omega_{1}, \forall t \in S, \forall u \in \mathbf{Q}^{+}, \quad V(X(\omega), t, u)=v(t, u)
$$

où

$$
\begin{aligned}
v(t, u)=\lim _{\varepsilon \rightarrow 0} \mathbf{E}\left[\operatorname { s u p } \left\{N\left[X(s)-X\left(s^{\prime}\right)\right] ;|t-s| \vee\left|t-s^{\prime}\right| \leq u,\right.\right. \\
\\
\left.\left.\left|s-s^{\prime}\right| \leq \varepsilon,\left\{s, s^{\prime}\right\} \subset S\right\}\right] .
\end{aligned}
$$

L'implication évidente

$$
|t-s| \leq h \Rightarrow V(f, t, u) \leq V(f, s, u+h), v(t, u) \leq v(s, u+h)
$$

et la densité de $S$ montrent alors que

$$
\forall \omega \in \Omega_{1}, \forall u>0, \forall t \in \mathbf{R}^{d}, \quad v\left(t, u^{-}\right) \leq V(X(\omega), t, u) \leq v\left(t, u^{+}\right) ;
$$

on a donc

$$
\forall \omega \in \Omega_{1}, \forall t \in \mathbf{R}^{d}, \quad V(X(\omega), t)=\lim _{u \rightarrow 0} v(t, u) ;
$$

c'est le résultat du lemme.

Démonstration de la proposition 2.3. (a) Si la propriété (1.1.3) est vérifiée, alors $X$ a une modification à trajectoires continues; la propriété (2.3.1) en résulte.

(b) Inversement, supposons la propriété (2.3.1) vérifiée; fixons une seminorme continue $N$ sur $E$; avec les notations du lemme 2.3.2, l'hypothèse (2.3.1) implique que $w(0)=0$. Les propriétés de contraction des séries de Rademacher montrent que

$$
\forall(t, s) \subset \mathbf{R}^{d}, \quad w(t) \leq 2 w(s)
$$

On a donc pour tout $t \in \mathbf{R}^{d}, w(t)=0$. La compacité de $[0,1]^{d}$ montre alors que

$\forall \omega \in \Omega_{1}, \quad \lim _{\varepsilon \rightarrow 0} \sup \left\{N\left[X(\omega, s)-X\left(\omega, s^{\prime}\right)\right] ;\left|s-s^{\prime}\right| \leq \varepsilon,\left\{s, s^{\prime}\right\} \subset S_{0}\right\}=0$.

La topologie de $E$ étant engendrée par une famille dénombrable de seminormes, ceci implique qu'il existe un ensemble presque sûr $\Omega_{2}$ sur lequel la restriction $X^{\prime}(\omega)$ de $X(\omega)$ à $S_{0}$ est uniformément continue; pour tout $\omega \in$ $\Omega_{2}$, on peut donc prolonger $X^{\prime}(\omega)$ en une application continue de $[0,1]^{d}$ dans $E$. On pose alors $X^{\prime}(\omega)=0$ en dehors de $\Omega_{2}$; puisque $\Omega$ est complet, $X^{\prime}$ est une fonction aléatoire à valeurs dans $E$; c'est une modification de $X$ puisque cette dernière est continue en probabilité : $X$ a donc une modification à trajectoires contintues; la propriété (1.1.3) en résulte.

Propositron 2.4. Supposons que la propriété (1.1.3) ne soit pas vérifiée; alors la propriété suivante est vérifiée:

(2.4.1) Pour toute suite $\left(n_{k}\right)$ d'entiers croissant vers l'infini, on a

$$
\mathbf{P}\left\{\exists t \in[0,1]^{d}: \sum_{k=0}^{\infty}\left\{\sum X_{n}(t) ; n_{k} \leq n<n_{k+1}\right\} \text { diverge }\right\}=1 .
$$


Démonstration. Puisque la propriété (1.1.3) n'est pas vérifiée, la proposition 2.3 montre qu'il existe une semi-norme continue non aléatoire $N$ sur $E$ et une variable aléatoire $\lambda>0$ telles que

$$
\begin{aligned}
\mathbf{P}\left\{\limsup _{t \rightarrow 0}[N[X(t)\right. & \left.\left.-X(0)] ; t \in \mathbb{Q}^{d}\right]=\lambda\right\} \\
& =\mathbf{P}\left\{\underset{t \rightarrow 0}{\lim \sup }\left[N\left[R_{k}(t)-R_{k}(0)\right] ; t \in Q^{d}\right]=\lambda\right\}=1,
\end{aligned}
$$

de sorte que $\lambda$ est une variable aléatoire terminale pour la suite indépendante $\left(\varepsilon_{n}\right)$ et elle est p.s. non aléatoire. Il existe alors un ensemble presque sûr $\Omega_{0}$ tel que par la propriété (2.3.3),

$\forall \omega \in \Omega_{0}, \forall t \in \mathbf{Q}^{d}, \forall k \in \mathrm{N}, \quad \lim _{s \rightarrow t, s \in \mathbf{R}^{d}} \sup _{l>k} N\left[\sum_{n=k}^{l}\left(X_{n}(t)-X_{n}(s)\right)\right]>\lambda / 4$

pour tout $\omega \in \Omega_{0}$ et tout $k \in N$, nous posons

$$
U_{k}(\omega)=\left\{t \in[0,1]^{d}: \sup _{l>k} N\left[\sum_{n=k}^{l} X_{n}(t)\right]>\lambda / 8\right\}
$$

alors $U_{k}(\omega)$ est un ouvert partout dense de $[0,1]^{d}$ et le théorème de Baire indique que l'intersection des $U_{k}(\omega), k \in \mathbf{N}$, n'est pas vide; soit $t=t(\omega)$ un élément de cette intersection; alors la série $\sum X_{n}(\omega, t)$ diverge. C'est le résultat énoncé si $\left(n_{k}\right)=\mathrm{N}$. Le résultat général s'établit de la même façon.

\section{Démonstration du théorème}

3.1. Suppposons pour commencer que la suite positive $\left(\lambda_{n}\right)$ soit non bornée et que $E$ contienne $c_{0}$; on peut alors construire une suite strictement croissante $\left(n_{j}\right)$ d'entiers positifs et une suite $\left(\lambda_{j}^{\prime}\right)$ extraite de la suite $\left(\lambda_{n}\right)$ telle que

$$
\forall j \in \mathbf{N}, \quad 2^{n_{j}} \leq \lambda_{j}^{\prime} \leq 2^{n_{j}+1}, \quad n_{j} \geq j .
$$

Nous notons $\left(\varepsilon_{m, j}\right)$ une suite double de Rademacher et nous posons dans ces conditions, en suivant un schéma proche de celui de [5], 13.16,

$$
F_{m}(t)=m^{-1} \sum_{j=m+1}^{2 m} \varepsilon_{m, j} \exp \left[i \lambda_{j}^{\prime} t\right], \quad m \geq 1, t \in \mathbb{R}
$$

on a donc

(3.1.3) $\quad \forall t \in \mathbf{R}, \forall m \geq 1, \quad\left|F_{m}(t)\right| \leq 1, \quad \mathbf{E}\left|F_{m}(t)\right|^{2}=m^{-1}$.

Les propriétés (3.1.1) et celles des séries trigonométriques lacunaires montrent alors qu'il existe des nombres $C_{1}>0$ et $C_{2}<\infty$ indépendants de la suite $\left(\lambda_{n}\right)$ tels que, puisque (3.1.2) implique que $2^{-m} \lambda_{m}^{\prime} \geq 1$,

$$
\forall m \geq 1, \quad C_{1} \leq \mathbf{E} \sup _{t \in\left[0,2^{-m}\right]}\left|F_{m}(t)\right| \leq \mathbf{E} \sup _{t \in[0,1]}\left|F_{m}(t)\right| \leq C_{2} .
$$

On pose $F:=\left\{F_{m}(t) ; m \in \mathbb{N}, t \in \mathbf{R}\right\}$. La propriété (3.1.3) et les inégalités de Khinchin montrent que pour tout $t \in \mathbf{R}$, la série $\sum\left|F_{m}(t)\right|^{4}$ est p.s. convergente; il en résulte que

$$
\forall t \in \mathbf{R}, \quad \mathbf{P}\left\{F(t) \in c_{0}\right\}=1 ;
$$

la même propriété (3.1.3) montre aussi que

$$
\forall t \in \mathbf{Q}, \quad \sup _{m \geq 1}\left|F_{m}(t)\right| \leq 1
$$

le théorème 4.1 .9 de [2] implique donc que $F$ a une modification à valeurs dans $c_{0}$ et à trajectoires bornées. Par contre, la propriété (3.1.4) montre que

$$
\begin{aligned}
\forall m \geq 1, \quad \mathrm{E} \sup _{t \in\left[0,2^{-m}\right]}\|F(t)-F(0)\|_{c_{0}} & \geq \mathrm{E} \sup _{t \in\left[0,2^{-m}\right]}\left|F_{m}(t)\right|-\mathbf{E}\left|F_{m}(0)\right| \\
& \geq C_{1}-m^{-1 / 2},
\end{aligned}
$$

donc

$$
\lim _{m \rightarrow \infty} \mathbf{E} \sup _{t \in\left[0,2^{-m}\right]}\|F(t)-F(0)\|_{c_{0}} \geq C_{1}
$$

de sorte que les propriétés d'intégrabilité des sornmes de Rademacher impliquent que $F$ n'a pas de modification à trajectoires continues dans $c_{0}$.

Par ailleurs, puisque $E$ contient $c_{0}$, il existe une suite $\left(x_{n}\right)$ contenue dans $E$ vérifiant les propriétés suivantes :

(3.1.6) $\forall\left(f_{n}\right) \in c_{0}, \sum f_{n} x_{n}$ converge dans $E$,

(3.1.7) l'ensemble $\left\{\sum_{n=1}^{N} f_{n} x_{n} ;\left\|f_{n}\right\|_{\infty} \leq 1, N \in \mathbf{N}\right\}$ est borné dans $E$,

(3.1.8) il existe une semi-norme continue $N$ sur $E$ telle que

$$
\inf _{n \in N} N\left(x_{n}\right) \geq 1 \text {. }
$$

\section{Posons alors pour tout $n \in \mathrm{N}$,}

$$
X_{n}=\left\{\begin{array}{l}
0 \text { si } \lambda_{n} \text { n'appartient pas à l'ensemble }\left(\lambda_{j}^{\prime}, j \in \mathbf{N}\right), \\
\sum\left\{\varepsilon_{m, j} m^{-1} x_{m} ; m \in\left[j / 2, j[\} \text {. si } \lambda_{n}=\lambda_{j}^{\prime},\right.\right.
\end{array}\right.
$$

et formons finalement les trois séries :

$$
\begin{aligned}
& S_{1}=\sum F_{n} x_{n}, \quad S_{2}=\sum_{m=1}^{\infty} \sum_{j=m+1}^{2 m} \varepsilon_{m, j} m^{-1} x_{m} \exp \left[i \lambda_{j}^{\prime} t\right], \\
& S_{3}=\sum X_{n} \exp \left[i \lambda_{n} t\right] .
\end{aligned}
$$


Dans ces conditions, puisque $F$ a une modification bornée, la série $S_{1}$ a p.s. des sommes partielles uniformément bornées dans $E$. C'est une série extraite de la série à termes symétriques $S_{2}$; les inégalités de Lévy montrent donc que les sommes partielles de la série $S_{2}$ ont la même propriété et aussi les sommes partielles de la série $S_{3}$ extraites de la série $S_{2}$ : ceci montre donc que la série $S_{3}$ vérifie la propriété (1.1.2). Par contre, la série $S_{3}$ ne vérifie pas la propriété (1.1.3); sinon les inégalités de Lévy montreraient que la série $S_{2}$ à termes symétriques dont elle est extraite convergerait aussi localement uniformément presque sûrement; la série $S_{1}$ extraite aurait la même propriété et ceci est contradictoire avec les propriétés (3.1.5) et (3.1.8) qui impliquent que le terme général de cette dernière série ne converge pas uniformément presque sûrement vers zéro au voisinage de $t=0$. Cette construction justifie donc l'énoncé (a) du théorème.

3.2. Supposons maintenant que $E$ ne contienne pas $c_{0}$ et que la série $\sum_{n=1}^{\infty} X_{n} \exp i\left\langle\lambda_{n}, t\right\rangle$ vérifie la propriété (1.1.2) sans vérifier la propriété (1.1.3); alors la symétrie de la famille $\left(X_{n}\right)$ montre qu'il existe une suite de variables de Rademacher indépendantes $\left(\varepsilon_{n}\right)$ et un ensemble presque sûr $\Omega_{0}$ tels que pour tout $\omega \in \Omega_{0}$, la série $\sum_{n=1}^{\infty} X_{n}(\omega) \varepsilon_{n} \exp i\left\langle\lambda_{n}, t\right\rangle$ vérifie aussi la propriété (1.1.2) sans vérifier la propriété (1.1.3). Dans ces conditions, la proposition 2.3 montre alors que, $\omega$ étant ainsi fixé, pour toute suite $\left(n_{k}\right)$ d'entiers croissant vers l'infini,

$$
\begin{aligned}
& \mathbf{P}\left\{\exists t \in[0,1]^{d}: \sum_{k=0}^{\infty}\left\{\sum X_{n}(\omega) \varepsilon_{n} \exp i\left\langle\lambda_{n}, t\right\rangle ;\right.\right. \\
&\left.\left.n_{k} \leq n<n_{k+1}\right\} \text { diverge }\right\}=1,
\end{aligned}
$$

alors que la proposition 2.4 implique la conclusion opposée, il y a donc contradiction : nécessairement la série $\sum_{n=1}^{\infty} X_{n} \exp i\left\langle\lambda_{n}, t\right\rangle$ vérifie la propriété (1.1.3); le théorème est démontré.

3.3. Preuve du corollaire. Sous les hypothèses du corollaire, notons $\left(X_{n}^{\prime}\right)$ une copie indépendante de la suite $\left(X_{n}\right)$ et $\left(U_{n}\right)=\left(X_{n}-X_{n}^{\prime}\right)$; alors la suite $\left(U_{n}\right)$ est symétrique et le théorème montre que

$$
\mathbf{P}\left\{\sum_{n=1}^{\infty} U_{n} \exp i\left\langle\lambda_{n}, t\right\rangle, t \in[0,1]^{d} \text { converge uniformément dans } E\right\}=1 ;
$$

le théorème de Fubini fournit donc la conclusion du corollaire.

\section{Références}

[1] V. V. Buldygin and S. A. Solntsev, Equivalence of sample and sequential continuity of gaussian processes and the continuity of gaussion Markov processes, Theory Probab. Appl. 33 (1988), 624-637.
[2] X. F ernique, Fonctions aléatoires à valeurs dans les. espaces lusiniens, Expositiones Math. 8 (1990), 289-364.

[3] -, Analyse de fonctions aléatoires gaussiennes stationnaires à valeurs vectorielles, Technical Report 331, Center of Stochastic Processes, University of N.C. at Chapel Hill, 1991.

$\left[3^{\prime}\right] \quad-$, Analyse de fonctions aléatoires gaussiennes stationnaires à valeurs vectorielles, C. R. Acad. Sci. Paris Sér. I 312 (1991), 927-929.

[4] J. Hoff $\mathrm{mann}-\mathrm{J} \emptyset \mathrm{rgensen}$, Sums of independent Banach space valued random variables, Studia Math. 52 (1974), 159-186.

[5] M. Ledoux and M. Talagrand, Probability in Banach Spaces, Ergeb. Math. (3) 23, Springer, Berlin 1991.

[6] S. K wapień, On Banach spaces containing $c_{0}$, Studia Math. 52 (1974), 187-188.

[7] M. B. Marcus and G. Pisier, Random Fourier series with applications to harmonic analysis, Ann. of Math. Stud. 101, Princeton Univ. Press, 1981.

[8] M. Talagrand, On vector valued random Fourier series, preprint 1991.

\section{DÉPARTEMENT DE MATHÉMATIQUE}

INSTITUT DE RECHERCHE MATHÉMATIQUE AVANCÉE

UNIVERSITE LOUIS PASTEUR

7, RUE RENE DESCARTES

67084 STRASBOURG CEDEX, FRANCE 